\title{
Cost-effectiveness of problem-solving treatment in comparison with usual care for primary care patients with mental health problems: a randomized trial
}

\author{
Judith E Bosmans ${ }^{1 *}$, Bettine Schreuders ${ }^{2}$, Harm WJ van Marwijk², Jan H Smit ${ }^{3}$, Patricia van Oppen ${ }^{2,3}$ \\ and Maurits W van Tulder ${ }^{1,4}$
}

\begin{abstract}
Background: Mental health problems are common and are associated with increased disability and health care costs. Problem-Solving Treatment (PST) delivered to these patients by nurses in primary care might be efficient. The aim of this study was to evaluate the cost-effectiveness of PST by mental health nurses compared with usual care (UC) by the general practitioner for primary care patients with mental health problems.

Methods: An economic evaluation from a societal perspective was performed alongside a randomized clinical trial. Patients with a positive General Health Questionnaire score (score $\geq 4$ ) and who visited their general practitioner at least three times during the past 6 months were eligible. Outcome measures were improvement on the Hospital Anxiety and Depression Scale and QALYs based on the EQ-5D. Resource use was measured using a validated questionnaire. Missing cost and effect data were imputed using multiple imputation techniques. Bootstrapping was used to analyze costs and cost-effectiveness of PST compared with UC.
\end{abstract}

Results: There were no statistically significant differences in clinical outcomes at 9 months. Mean total costs were $€ 4795$ in the PST group and €6857 in the UC group. Costs were not statistically significantly different between the two groups (95\% Cl -4698;359). The cost-effectiveness analysis showed that PST was cost-effective in comparison with UC. Sensitivity analyses confirmed these findings.

Conclusions: PST delivered by nurses seems cost-effective in comparison with UC. However, these results should be interpreted with caution, since the difference in total costs was mainly caused by 3 outliers with extremely high indirect costs in the UC group.

Trial registration: Nederlands Trial Register ISRCTN51021015

Keywords: Costs and cost analysis, Problem-solving treatment, Nurses, Depression, Anxiety, Primary health care

\section{Background}

Mental health problems are prevalent, with depression and anxiety being most common [1-3]. The disability burden of mental health problems is enormous [3]. Moreover, mental health problems are associated with high health care costs and lost productivity costs, thus causing a huge economic burden as well [4-6].

\footnotetext{
*Correspondence: j.e.bosmans@vu.nl

'Department of Health Sciences and EMGO Institute for Health and Care Research, Faculty of Earth and Life Sciences, VU University Amsterdam, Amsterdam, The Netherlands

Full list of author information is available at the end of the article
}

Most patients with mental health problems receive primary care [7]. The perceived burden on general practitioners (GPs) when treating these patients is higher than when treating other patients [8]. Treatment mainly consists of psychotropic drugs [7,9], although these drugs have disadvantages such as side effects, dependency and poor compliance. Moreover, many patients prefer psychological treatments over psychotropic drugs and advice $[10,11]$. However, most GPs have little experience in delivering psychological interventions and there is little evidence on the effectiveness of psychological 
treatments delivered by GPs [12]. Furthermore, it is questionable whether busy GPs have time to provide psychological treatment to patients with mental health problems. Psychological treatments could be made more widely available, if other health care providers could provide these treatments in an integrated primary care setting.

Problem-Solving Treatment (PST) is a psychological treatment that has been shown to be effective for depression in primary care and that can be effectively delivered by nurses or GPs $[13,14]$. However, for unselected patients with mental health problems who constitute the bulk of the primary care caseload, evidence of the effectiveness of PST was less clear and PST was associated with significantly higher health care costs than usual GP care $[15,16]$. The aim of this study was to assess the cost-effectiveness of PST by mental health nurses compared with usual GP care for primary care patients with mental health problems in The Netherlands.

\section{Methods}

This economic evaluation was conducted alongside a randomized controlled trial with nine month follow-up that was performed in 12 general practices in and around Amsterdam, The Netherlands, between November 2003 and May 2005. The Medical Ethical Committee of the University Medical Center in Amsterdam approved the study protocol. Block randomization was used to allocate patients to either PST provided by a mental health care nurse or usual care from the GP. All patients gave written informed consent before randomization. The methodological details of the trial and the three months effectiveness results are reported in detail elsewhere $[17,18]$.

\section{Patient selection}

Consecutive patients who visited their GP were invited to complete the General Health Questionnaire (GHQ12) [19]. Patients were eligible for the trial if they had a score of 4 points or more on the GHQ-12 [15,20], and visited their GP three times or more in the previous six months. Patients had to be 18 years or older, able to speak Dutch, and willing to undergo brief psychological treatment. Exclusion criteria were the existence of potentially life-threatening somatic and mental disorders, the existence of somatic and mental disorders limiting the patient's ability to participate or adhere to treatment, and mental health treatment during the previous year.

\section{Usual care}

Treatment of mental health problems in the usual care (UC) group was not restricted in any way. Dutch GPs are encouraged to work according to guidelines issued by the Dutch College of GPs [21,22]. The depression guidelines recommend that treatment of depression primarily consists of education and coaching. Antidepressant treatment and/or referral for psychotherapy can be added, depending on the duration and severity of the depressive symptoms, the limitations in daily functioning, and the patient's preference [22]. For anxiety, the guidelines recommend referral for psychotherapy and medication if indicated [21].

\section{PST}

PST is a brief treatment focused on practical skill building, education, and managing depressive symptoms. The goal is to stimulate an active attitude towards everyday problems and, hereby, to achieve a reduction in mental health problems. Twelve nurses working at one of the mental healthcare organizations in Amsterdam were trained during two days by two researchers who developed PST for primary care, L. Mynors-Wallis and I. Davies. In the second part of the training, the nurses treated four patients closely supervised by a cognitive behavioral therapist (PvO). The supervisor was certified as supervisor by the National Association of Behavior Therapy and Cognitive Therapy in The Netherlands.

Patients were offered four to six PST sessions. The first session lasted a maximum of 60 minutes and following sessions a maximum of 30 minutes. Patients could visit their GP for UC if necessary.

\section{Clinical outcome measures}

The participants received written questionnaires at baseline, and at three and nine months after baseline. The primary clinical outcome was severity of mental health symptoms measured using the Hospital Anxiety and Depression Scale (HADS) [23]. Lower scores on the HADS indicate less severe symptoms. Quality of life was measured using the EQ-5D [24]. The EQ-5D scores were used to calculate utilities using the Dutch tariff [25]. QALYs were calculated using linear interpolation between time points. Higher QALY scores indicate more improvement in quality of life.

\section{Cost measures}

Cost data were collected from a societal perspective 3 times during the 9 months follow-up using the TiC-P questionnaire with a recall period of 3 months [26]. If available, Dutch guideline prices were used to value resource use $[27,28]$. Medication was valued using prices of the Royal Dutch Society for Pharmacy [29]. Costs of visits to complementary therapists were based on prices from the therapists themselves. Lost productivity costs were calculated according to the friction cost approach (friction period 154 days) using the mean age- and sex- 
specific income of the Dutch population [28]. According to the friction cost approach a sick employee is replaced after a certain amount of time (the friction period) after which there are no lost productivity costs anymore. Table 1 lists the cost categories included in the economic evaluation and the prices used. All costs were adjusted to the year 2012 using consumer price indices [30].

\section{Analysis}

It was estimated that 65 patients in each group would be needed to detect an effect size of 0.4 (2-sided $\alpha=0.05$, $\beta=0.20$ ). Taking into account a drop-out rate of $20 \%$, the aim was to include 160 patients into the trial.

The statistical analyses were performed according to the intention-to-treat principle. Multiple Imputation (MI) according to the Multivariate Imputation by Chained Equations (MICE) algorithm was done to impute missing cost and effect data with SPSS 17.0 for Windows (SPSS Inc., Chicago, IL, USA). Predictive mean matching was used to account for the skewness of the cost data and the fact that costs are bounded by zero. An imputation model was constructed that included variables that were related to missingness or predicted the outcome variable. By MI 5 imputed data sets were created, each of which was analyzed separately. The results of the 5 analyses were pooled using Rubin's rules [31].

Linear regression was used to estimate differences in costs and effects. Costs generally have a highly skewed distribution. Therefore, bootstrapping with 5000 replications was used to estimate "approximate bootstrap confidence" (ABC) intervals around cost differences [32,33].

Incremental cost-effectiveness ratios (ICERs) were calculated by dividing the difference in total costs between PST and UC by the difference in clinical effects. Nonparametric bootstrapping was also used to estimate the uncertainty surrounding the incremental costeffectiveness and cost-utility ratios (5000 replications). The bootstrapped cost-effect pairs were plotted on a cost-effectiveness plane [34] and used to estimate costeffectiveness acceptability curves. Cost-effectiveness acceptability curves show the probability that the intervention is cost-effective in comparison with the control treatment for a range of ceiling ratios. The ceiling ratio is defined as the amount of money society is willing to pay to gain one unit of effect [35].

Table 1 Health care utilization over 9 months of patients with complete cost data

\begin{tabular}{|c|c|c|c|c|}
\hline Category & Price $(€)$ & PST $(n=56)$ & Usual care $(n=65)$ & Difference \\
\hline \multicolumn{5}{|l|}{ Primary care } \\
\hline General practitioner [no contacts] & 23.53 & $4.1(3.3)$ & $4.8(4.5)$ & $-0.7(-2.1 ; 0.8)$ \\
\hline Physiotherapist [no contacts] & 26.50 & $5.5(10.3)$ & $6.0(11.0)$ & $-0.5(-4.4 ; 3.4)$ \\
\hline Social worker [no contacts] & 55.75 & $0.3(1.9)$ & $0.2(0.8)$ & $0.1(-0.5 ; 0.5)$ \\
\hline Psychologist [no contacts] & 88.53 & $1.1(5.0)$ & $1.0(2.7)$ & $0.1(-1.3 ; 1.6)$ \\
\hline \multicolumn{5}{|l|}{ Secondary care } \\
\hline Outpatient clinic [no contacts] & 65.23 & $2.3(3.4)$ & $4.2(10.2)$ & $-1.9(-4.6 ; 0.8)$ \\
\hline Regional institute for mental welfare [no contacts] & 144.44 & $0.3(0.9)$ & $0.5(1.9)$ & $-0.2(-0.8 ; 0.3)$ \\
\hline In-hospital psychiatrist [no contacts] & $73.88-131.94^{*}$ & $0.1(0.7)$ & $0.2(0.9)$ & $-0.1(-0.4 ; 0.2)$ \\
\hline Day treatment [no half days] & $104.28-186.21^{*}$ & $0(0)$ & $0(0)$ & - \\
\hline Hospital admission [no days] & $291.21-554.45^{\dagger}$ & $0.3(1.1)$ & $1.3(5.8)$ & $-1.0(-2.4 ; 0.5)$ \\
\hline Home care [no hours] & 35.76 & $4.2(16.0)$ & $7.9(29.4)$ & $-3.7(-12.4 ; 5.0)$ \\
\hline Complementary therapists [no contacts] & $26.79-143.28^{+}$ & $1.4(3.8)$ & $1.8(4.8)$ & $-0.4(-1.9 ; 1.2)$ \\
\hline Occupational physician [no contacts] & 24.75 & $0.4(1.4)$ & $0.8(1.6)$ & $-0.4(-0.9 ; 0.2)$ \\
\hline Medication [no deliveries] & depending on type and dose & $7.0(6.8)$ & $7.4(6.5)$ & $-0.4(-2.8 ; 2.0)$ \\
\hline PST [no contacts] & $56.87^{\S}$ & $4.1(2.1)$ & - & \\
\hline Help from family/friends or paid help [no hours] & 10.36 & $14.9(32.8)$ & $12.5(34.2)$ & $2.4(-9.8 ; 14.5)$ \\
\hline Absenteeism [no days] & depending on age and sexe & $19.5(54.8)$ & $29.0(62.4)$ & $-9.5(-30.8 ; 11.8)$ \\
\hline Presenteeism [no days] & depending on age and sexe & $3.8(12.9)$ & $7.2(19.9)$ & $-3.4(-10.0 ; 2.7)$ \\
\hline
\end{tabular}

Presented are means (SD) and mean differences $(95 \% \mathrm{CI})$.

PST = Problem-Solving Treatment.

* depending on type of hospital: academic, general or psychiatric hospital.

${ }^{+}$depending on type of hospital: academic, general, rehabilitation or psychiatric hospital.

* depending on type of therapist.

$\S$ Total PST costs based on 6 sessions: $€ 341.24$ (training $€ 45.08$, treatment $€ 244.24$, housing $€ 51.92$ ). 


\section{Sensitivity analyses}

Six sensitivity analyses were performed to assess the robustness of the results. In the first sensitivity analysis, statistical analysis was restricted to patients with complete follow-up data. Secondly, we redid the economic evaluation from a NHS perspective meaning that only direct costs were included in the analyses. In the third sensitivity analysis, lost productivity costs were calculated according to the human capital approach. This approach assumes that lost productivity costs are generated until an employee recovers and returns to work, or until the moment of death or retirement. In the fourth sensitivity analysis, training costs were excluded from the PST intervention costs. The fifth sensitivity analysis concerned a per protocol analysis in which only PST patients were included who completed 4 or more PST sessions. Finally, in the sixth sensitivity analysis the observed outliers with very high lost productivity costs were excluded from the analysis.

\section{Results}

During the inclusion period, 2133 patients completed the GHQ-12 and 353 refused to participate. 622 patients had a score of 4 or more on the GHQ-12 and visited their GP three times or more in the past 6 months. 311 patients were unwilling to participate in the trial and 136 did not meet the inclusion criteria, leaving 175 patients to be included in the trial of whom 88 were randomized to the PST group and 87 to the UC group.

At baseline, utility was significantly lower in UC patients than in PST patients meaning that the health status of UC patients was lower than of PST patients. All analyses concerning QALYs were corrected for this baseline difference. There were no other significant differences in clinical and demographic characteristics between the treatment groups at baseline (Table 2).

Table 2 Baseline characteristics

\begin{tabular}{lcc}
\hline & PST (n=88) & Usual care (n=87) \\
\hline Mean (SD) age (years) & $52(16)$ & $53(16)$ \\
Female & $68(77 \%)$ & $57(66 \%)$ \\
Married/cohabiting & $34(39 \%)$ & $33(38 \%)$ \\
Education level & & \\
Low & $17(19 \%)$ & $14(16 \%)$ \\
Medium & $22(25 \%)$ & $23(26 \%)$ \\
High & $31(35 \%)$ & $32(37 \%)$ \\
Unknown & $18(21 \%)$ & $60(69 \%)$ \\
Born in The Netherlands & $62(71 \%)$ & $0.62(0.28)$ \\
Mean EuroQol utility (SD) & $0.73(0.18)$ & $16.9(7.1)$ \\
Mean HADS score (SD) & $15.4(7.5)$ & \\
\hline
\end{tabular}

Presented are numbers (\%) unless stated otherwise. HADS $=$ Hospital Anxiety Depression Scale.
Complete cost data were available for $56(64 \%)$ of the PST patients and $65(75 \%)$ of the UC patients. There were no significant differences in baseline characteristics between patients with and without complete cost data.

\section{Clinical effects}

Clinical effects are presented in Table 3. After 9 months the multiply imputed pooled improvement in HADS score was -3.1 (SE 1.3) in PST patients and -2.9 (SE 1.0) in UC patients indicating that PST patients improved more than UC patients. However, this difference was not statistically significant (mean difference -0.2 , 95\% CI -3.7; 3.2).

The mean pooled utility score based on the Dutch tariff for the EQ-5D was 0.71 in the PST group and 0.61 in the UC group. After 3 and 9 months the pooled utility scores were 0.76 and 0.73 , respectively, in the PST group and 0.66 and 0.70 , respectively, in the UC group. The mean number of QALYs after 9 months was 0.56 (SE 0.02) in the PST group and 0.50 (SE 0.02) in the UC group. After correction for baseline utility, the difference in QALYs between PST and UC was in favour of the PST group (0.03), but statistically non-significant ( $95 \%$ CI $-0.02 ; 0.08)$.

\section{Health care utilization}

Table 1 presents the health care utilization in the PST and the UC groups at 9 months for patients with complete cost data. In general, health care utilization in the PST group was slightly lower than in the UC group for both general and mental health care.

Table 3 Multiply imputed pooled outcomes and costs over 9 months

\begin{tabular}{lccc}
\hline Cost category & $\begin{array}{c}\text { PST } \\
(\mathbf{n = 8 8})\end{array}$ & $\begin{array}{c}\text { Usual care } \\
(\mathbf{n}=\mathbf{8 7})\end{array}$ & Difference \\
\hline Outcome & & & \\
HADS & $-3.1(1.3)$ & $-2.9(1.0)$ & $-0.2(-3.7 ; 3.2)$ \\
QALY & $0.56(0.02)$ & $0.49(0.02)$ & $0.03(-0.02 ; 0.08)^{*}$
\end{tabular}

\section{Cost category}

\begin{tabular}{|c|c|c|c|}
\hline Direct costs & $1751(221)$ & $2004(340)$ & $-253(-1149 ; 476)$ \\
\hline $\begin{array}{l}\text { Direct healthcare } \\
\text { costs }\end{array}$ & $1362(202)$ & $1829(326)$ & $-467(-1340 ; 202)$ \\
\hline $\begin{array}{l}\text { Direct non-healthcare } \\
\text { costs }\end{array}$ & $214(46)$ & $175(56)$ & $40(-117 ; 166)$ \\
\hline PST costs & $174(15)$ & $0(0)$ & $174(143 ; 206)$ \\
\hline Indirect costs & $3768(703)$ & $5889(1187)$ & $-2121(-4788 ; 396)$ \\
\hline Costs absenteeism & $2991(708)$ & $4319(951)$ & $-1328(-3748 ; 823)$ \\
\hline sts presenteeism & $778(251)$ & $1570(581)$ & $-792(-2343 ; 209)$ \\
\hline tal costs & $4795(671)$ & $6857(1128)$ & $-2062(-4698 ; 359)$ \\
\hline
\end{tabular}

Presented are means (SEs) and mean differences (95\% Cls).

PST = Problem-Solving Treatment; HADS = Hospital Anxiety Depression Scale; QALY $=$ Quality-Adjusted Life-Years.

* corrected for baseline utility. 
Sixty-four (73\%) PST patients received at least 1 PST session. On average, PST patients received 3 PST sessions. During the study period, 51 (91\%) PST patients and $57(88 \%)$ UC patients visited the general practitioner at least once. Thirty-two (36\%) PST patients received some form of mental health care (referral to a mental health care provider or prescription of either an antidepressant or benzodiazepine) in comparison with 33 (38\%) UC patients during follow-up. Absenteeism in the UC group was substantially higher than in the PST group. There were $2(4 \%)$ patients in the PST group and 4 (6\%) patients in the UC group who were absent for more than 180 days.

\section{Costs}

Table 3 presents the imputed pooled mean total costs in the PST and UC group after 9 months. Both direct and indirect costs in the PST group were lower than in the UC group. Indirect costs were the greatest contributor to mean total costs. Mean PST costs amounted to $€ 174$ (SE 15). Mean total costs in the PST group were $€ 2062$ lower than in the UC group, but this difference was not statistically significant (95\% CI -4698; 359).

Total mental health care costs were $€ 485$ in the PST group and $€ 259$ in the UC group. Although this difference was considerable, it did not reach statistical significance (mean difference $€ 226,95 \%$ CI -3; 448).

\section{Cost-effectiveness}

The results of the cost-effectiveness and cost-utility analyses are presented in Table 4. The ICER for improvement in total HADS score at 9 months was 8676, meaning that one point improvement extra in the PST group costs $€ 8676$ extra in comparison with the UC group. In the CE plane, only $5 \%$ of the costeffect pairs was located in the NE and NW quadrants, while $55 \%$ and $40 \%$ of the cost-effect pairs was located in the SE and SW quadrant, respectively. The costeffectiveness acceptability curve in Figure 1 shows that for a ceiling ratio of $€ 0$ per point of improvement on the HADS, the probability that PST is cost-effective in comparison with UC is 0.95 . For higher ceiling ratios this probability decreases slowly to 0.57 .

The difference in QALYs after 9 months between the PST and UC groups was very small leading to a very large ICER. The CE plane showed that $88 \%$ and $7 \%$ of the cost-effect pairs was located in the SE and SW quadrants, respectively. The cost-effectiveness acceptability curve in Figure 2 shows that PST is considered costeffective in comparison with UC for all ceiling ratios.

\section{Sensitivity analyses}

Results of the sensitivity analyses are presented in Table 4. PST was considered cost-effective in comparison with UC in all sensitivity analyses. In the complete case analyses, differences in clinical effects were small and not statistically significant. Total costs in the PST group were significantly lower than in the UC group. Therefore, PST was considered cost-effective in comparison with UC in the complete case analyses.

In the sensitivity analysis performed from the NHS perspective, costs in the PST group were $€ 253$ lower

Table 4 Results of cost-effectiveness and cost-utility analyses

\begin{tabular}{|c|c|c|c|c|c|c|c|c|c|c|}
\hline \multirow[t]{2}{*}{ Analysis } & \multicolumn{2}{|c|}{ Sample size } & \multirow[t]{2}{*}{ Outcome } & \multirow{2}{*}{$\frac{\text { Cost difference }(€)}{(95 \% \mathrm{Cl})}$} & \multirow{2}{*}{$\frac{\text { Effect difference }}{(95 \% \mathrm{Cl})}$} & \multirow[t]{2}{*}{ ICER } & \multicolumn{4}{|c|}{ Distribution CE plane } \\
\hline & PST & UC & & & & & NE & SE & SW & NW \\
\hline \multirow[t]{2}{*}{ Multiply imputed analysis } & 88 & 87 & HADS & $-2062(-4698 ; 359)$ & $-0.2(-3.7 ; 3.2)$ & 8676 & $2 \%$ & $55 \%$ & $40 \%$ & $3 \%$ \\
\hline & 88 & 87 & QALY* & $-2062(-4698 ; 359)$ & $0.03(-0.02 ; 0.08)$ & -65045 & $4 \%$ & $88 \%$ & $7 \%$ & $1 \%$ \\
\hline \multirow[t]{2}{*}{ Complete cases } & 56 & 63 & HADS & $-2715(-5858 ; 77)$ & $0.02(-2.3 ; 2.4)$ & -113112 & $1 \%$ & $48 \%$ & $49 \%$ & $2 \%$ \\
\hline & 53 & 59 & QALY* & $-3085(-6300 ;-171)$ & $0.04(-0.01 ; 0.08)$ & -83380 & $2 \%$ & $94 \%$ & $4 \%$ & $0 \%$ \\
\hline \multirow[t]{2}{*}{ NHS perspective ${ }^{\dagger}$} & 88 & 87 & HADS & $-253(-1149 ; 476)$ & $-0.2(-3.7 ; 3.2)$ & 1065 & $11 \%$ & $45 \%$ & $28 \%$ & $16 \%$ \\
\hline & 88 & 87 & QALY* & $-253(-1149 ; 476)$ & $0.03(-0.02 ; 0.08)$ & -7984 & $23 \%$ & $69 \%$ & $4 \%$ & $4 \%$ \\
\hline \multirow[t]{2}{*}{ Human capital approach } & 88 & 87 & HADS & $-2397(-5427 ; 393)$ & $-0.2(-3.7 ; 3.2)$ & 10084 & $2 \%$ & $55 \%$ & $40 \%$ & $3 \%$ \\
\hline & 88 & 87 & QALY* & $-2397(-5427 ; 393)$ & $0.03(-0.02 ; 0.08)$ & -75597 & $4 \%$ & $88 \%$ & $7 \%$ & $1 \%$ \\
\hline \multirow[t]{2}{*}{ Training costs PST excluded } & 88 & 87 & HADS & $-2035(5669 ; 1259)$ & $-0.2(-3.7 ; 3.2)$ & 8564 & $6 \%$ & $50 \%$ & $38 \%$ & $6 \%$ \\
\hline & 88 & 87 & QALY* & $-2035(5669 ; 1259)$ & $0.03(-0.02 ; 0.08)$ & -64198 & $11 \%$ & $81 \%$ & $7 \%$ & $1 \%$ \\
\hline \multirow[t]{2}{*}{ Per protocol analysis } & 41 & 87 & HADS & $-3220(-5744 ;-725)$ & $1.5(-1.5 ; 4.4)$ & -2943 & $0 \%$ & $16 \%$ & $84 \%$ & $0 \%$ \\
\hline & 41 & 87 & QALY* & $-3220(-5744 ;-725)$ & $0.03(-0.03 ; 0.09)$ & -137279 & $0 \%$ & $90 \%$ & $10 \%$ & $0 \%$ \\
\hline \multirow[t]{2}{*}{ Observed outliers excluded } & 88 & 84 & HADS & $-1090(-3492 ; 1175)$ & $-0.3(-3.8 ; 3.3)$ & 4297 & $7 \%$ & $49 \%$ & $33 \%$ & $11 \%$ \\
\hline & 88 & 84 & QALY* & $-1090(-3492 ; 1175)$ & $0.03(-0.02 ; 0.08)$ & -34498 & $15 \%$ & $77 \%$ & $5 \%$ & $3 \%$ \\
\hline
\end{tabular}

HADS = Hospital Anxiety and Depression Scale; QALY = Quality-Adjusted Life-Year; NHS = National Health Service.

* adjusted for baseline utility score.

${ }^{\dagger}$ only direct costs included. 


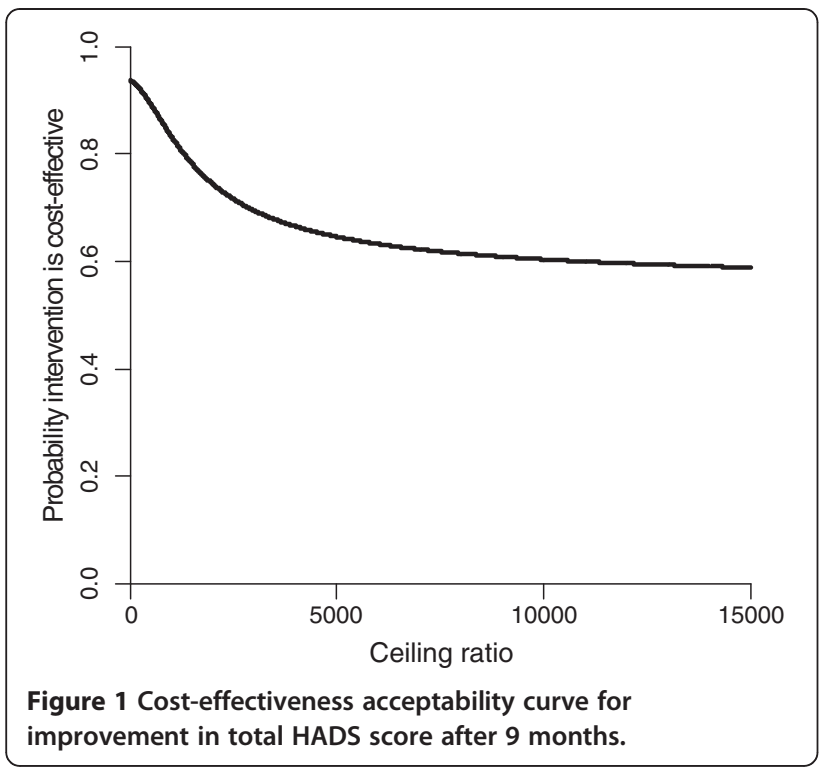

than in the UC group. This difference was statistically non-significant. CEA curves suggest that the willingness to pay should be high to consider PST cost-effective in comparison with UC (data not shown).

Use of the human capital approach led to higher estimates of indirect and total costs in both groups. However, the difference in total costs changed from $-€ 2062$ to $-€ 2397$ which was not statistically significant. In this analysis, PST was also considered cost-effective in comparison with UC.

Excluding training costs from the PST costs, resulted in lower costs for the PST treatment $(€ 151)$. The difference in total costs between PST and UC in this sensitivity analysis was $-€ 2035$, but this was not statistically

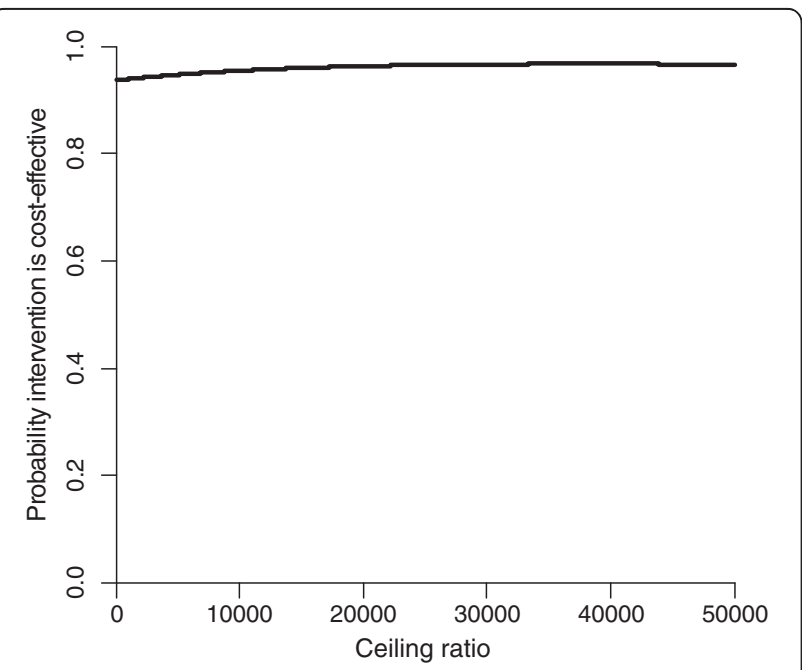

Figure 2 Cost-effectiveness acceptability curve for QALYs after 9 months. significant (95\% CI -5669; 1259). The conclusion regarding cost-effectiveness did not change in this analysis.

In the per protocol analysis, the difference in total costs was $-€-3220$ which was statistically significant (95\% CI -5744; -725). The differences in improvement in HADS score and QALY were somewhat larger than in the main analysis, but these differences were not statistically significant. PST was considered cost-effective in comparison with UC.

\section{Discussion}

Our results show that there were no significant differences in effects between PST and UC patients in this population of primary care patients with relatively mild levels of distress. The cost difference between PST and UC was substantial, but not statistically significant. Costeffectiveness planes and acceptability curves show that PST was cost-effective in comparison with UC. Indirect costs in the PST group were substantially lower than in the UC group and were the greatest contributor to the difference in total costs. However, this difference was mainly caused by 3 outliers in the UC group. Sensitivity analyses confirmed the small differences in effects and that costs in the PST group were substantially lower than in the UC group. Based on these analyses, PST was also considered cost-effective in comparison with UC in this study. Analyses from the NHS perspective suggested that PST is not considered cost-effective in comparison with UC.

UC patients had significantly lower utility scores at baseline than PST patients. Therefore, we suspect that morbidity rates in the UC group were higher than in the PST group. This may also explain the higher costs in the UC group. Additional analyses showed that the differences in direct and indirect costs were only partially explained by the difference in utility score at baseline (data not shown).

One of the strengths of this trial is that it was a pragmatic trial, meaning that we tried to resemble daily clinical practice as much as possible. By applying as few restrictions as possible on patient selection, we think we succeeded in recruiting a population that is representative of the patients with mental health problems seen by the GP. Also, we tried to model the GP's normal care process as much as possible. Therefore, the results of this study are likely to be generalisable to the rest of The Netherlands and other countries with similar health care systems.

Research suggests that a considerable part of lost productivity costs in mental disorders is caused by presenteeism [36,37]. Therefore, another important strength of this study is that both costs of absenteeism and presenteeism (being present at work, but at reduced work 
performance) were included, whereas earlier studies included only costs of absenteeism [15,16].

Our study also has some limitations. First, our study was underpowered to detect relevant cost differences which is reflected in the wide confidence intervals around the cost differences. This is a common problem in economic evaluations alongside clinical trials. Because of the heavily skewed distribution of cost data, very large numbers of patients are needed to detect relevant cost differences [38]. Second, the number of patients that did not return all cost questionnaires was considerable (PST group 36\%, UC group 25\%). However, there were no significant differences between patients with and without complete cost data, reducing the chance of bias caused by selective drop-out. Moreover, both the results of the imputed analysis and the complete case analysis showed that PST is cost-effective in comparison with UC. Finally, the follow-up period of the trial may have been too short. If PST indeed is beneficial in comparison with usual care, then it is reasonable to assume that these benefits extend over many years.

Our results can be compared with two other studies that included an economic evaluation of PST for primary care patients with mental health problems $[15,16]$. Our results are consistent with their findings that PST is not effective in comparison with usual GP care. These studies showed that PST was associated with higher costs than usual GP care, while in our study lower costs were found in the PST group. Possible explanations for this discrepancy include the length of the follow-up (9 months in this study versus 6 months in the other studies), the higher costs of the PST in the other studies, and the cost categories that were included.

The accompanying clinical trial showed in a post-hoc analysis that a sub-group of more severely depressed patients could benefit from PST [18]. This is in line with earlier research [13,14]. A recent review showed that PST is more effective in comparison with GP care for depression, and mixed anxiety and depression [39]. Thus, it is reasonable to assume that PST should be reserved for patients with more severe mental health symptoms. A potential fruitful avenue is the development of stepped care approaches in which PST is offered to patients who do not recover or deteriorate during a period of 'watchful waiting'.

\section{Conclusions}

In conclusion, this study shows that PST did not result in improved clinical outcomes in comparison with UC, but did result in substantially lower costs which are explained by the lower indirect costs in the PST group. Based on these results, PST was considered costeffective in comparison with usual care, although not from an NHS perspective. Since, most of the difference in costs in our study was caused by 3 outliers with very high lost productivity costs, a too strong conclusion on the cost-effectiveness of PST cannot be drawn based on the results of this study.

\section{Competing interests}

The authors declare that they have no competing interests.

\section{Authors' contributions}

PVO and HWJVM developed the design of the randomized clinical trial and participated in writing the article. BS collected the data. JEB analyzed the data and drafted the manuscript. All authors made substantial contributions to the interpretation of the data, were involved in drafting the manuscript, and gave final approval of the version to be published. All authors read and approved the final manuscript.

\section{Acknowledgements}

The Netherlands Organization for Health Research and Development (ZonMw) funded the trial, project number 4200.0001. ZonMw had no further role in study design; in the collection, analysis and interpretation of data; in the writing of the report; and in the decision to submit the paper for publication.

\section{Author details}

${ }^{1}$ Department of Health Sciences and EMGO Institute for Health and Care Research, Faculty of Earth and Life Sciences, VU University Amsterdam, Amsterdam, The Netherlands. ${ }^{2}$ Department of General Practice and EMGO Institute for Health and Care Research, VU University Medical Center, Amsterdam, The Netherlands. ${ }^{3}$ Department of Psychiatry and EMGO Institute for Health and Care Research, VU University Medical Center, Amsterdam, The Netherlands. ${ }^{4}$ Department of Epidemiology \& Biostatistics and EMGO Institute for Health and Care Research, VU University Medical Center, Amsterdam, The Netherlands.

Received: 4 April 2012 Accepted: 4 October 2012

Published: 10 October 2012

\section{References}

1. Ormel J, VonKorff M, Ustun TB, Pini S, Korten A, Oldehinkel T: Common mental disorders and disability across cultures. Results from the WHO Collaborative Study on Psychological Problems in General Health Care. JAMA 1994, 272(22):1741-1748.

2. Tiemens BG, Ormel J, Simon GE: Occurrence, recognition, and outcome of psychological disorders in primary care. Am J Psychiatry 1996, 153(5):636-644.

3. Wittchen HU, Jacobi F: Size and burden of mental disorders in Europe-a critical review and appraisal of 27 studies. Eur Neuropsychopharmacol 2005, 15(4):357-376.

4. Greenberg PE, Sisitsky T, Kessler RC, Finkelstein SN, Berndt ER, Davidson JR, Ballenger JC, Fyer AJ: The economic burden of anxiety disorders in the 1990s. J Clin Psychiatry 1999, 60(7):427-435.

5. Greenberg PE, Kessler RC, Birnbaum HG, Leong SA, Lowe SW, Berglund PA, Corey-Lisle PK: The economic burden of depression in the United States: how did it change between 1990 and 2000? J Clin Psychiatry 2003, 64(12):1465-1475.

6. Lecrubier $Y$ : The burden of depression and anxiety in general medicine. J Clin Psychiatry 2001, 62(Suppl 8):4-9.

7. Bushnell J, McLeod D, Dowell A, Salmond C, Ramage S, Collings S, Ellis P, Kljakovic M, McBain L: The treatment of common mental health problems in general practice. Fam Pract 2006, 23(1):53-59.

8. Zantinge EM, Verhaak PF, Kerssens JJ, Bensing JM: The workload of GPs: consultations of patients with psychological and somatic problems compared. Br J Gen Pract 2005, 55(517):609-614.

9. Donoghue JM, Tylee A: The treatment of depression: prescribing patterns of antidepressants in primary care in the UK. Br J Psychiatry 1996, 168(2):164-168

10. Priest RG, Vize C, Roberts A, Roberts M, Tylee A: Lay people's attitudes to treatment of depression: results of opinion poll for Defeat Depression Campaign just before its launch. BMJ 1996, 313(7061):858-859. 
11. van Schaik DJ, Klijn AF, van Hout HP, van Marwijk HW, Beekman AT, De Haan M, van Dyck R: Patients' preferences in the treatment of depressive disorder in primary care. Gen Hosp Psychiatry 2004, 26(3):184-189.

12. Huibers MJ, Beurskens AJ, Bleijenberg G, van Schayck CP: The effectiveness of psychosocial interventions delivered by general practitioners. Cochrane Database Syst Rev 2003, (2):CD003494.

13. Mynors-Wallis LM, Gath DH, Lloyd-Thomas AR, Tomlinson D: Randomised controlled trial comparing problem solving treatment with amitriptyline and placebo for major depression in primary care. BMJ 1995, 310(6977):441-445

14. Mynors-Wallis LM, Gath DH, Day A, Baker F: Randomised controlled trial of problem solving treatment, antidepressant medication, and combined treatment for major depression in primary care. BMJ 2000, 320(7226):26-30

15. Kendrick T, Simons L, Mynors-Wallis L, Gray A, Lathlean J, Pickering R, Harris S, Rivero-Arias O, Gerard K, Thompson C: Cost-effectiveness of referral for generic care or problem-solving treatment from community mental health nurses, compared with usual general practitioner care for common mental disorders: Randomised controlled trial. Br J Psychiatry 2006, 189:50-59.

16. Mynors-Wallis L, Davies I, Gray A, Barbour F, Gath D: A randomised controlled trial and cost analysis of problem-solving treatment for emotional disorders given by community nurses in primary care. $\mathrm{Br} \mathrm{J}$ Psychiatry 1997, 170:113-119.

17. Schreuders B, van Oppen P, van Marwijk HW, Smit JH, Stalman WA: Frequent attenders in general practice: problem solving treatment provided by nurses [ISRCTN51021015]. BMC Fam Pract 2005, 6:42.

18. Schreuders B, van Marwijk H, Smit J, Rijmen F, Stalman W, van Oppen P: Primary care patients with mental health problems: outcome of a randomised clinical trial. Br J Gen Pract 2007, 57(544):886-891.

19. Koeter M, Ormel J: General Health Questionnaire, Handleiding Nederlandse bewerking. Swets and Zeitlinger: Lisse; 1991.

20. Dowrick C, Dunn G, Ayuso-Mateos JL, Dalgard OS, Page H, Lehtinen V Casey P, Wilkinson C, Vazquez-Barquero JL, Wilkinson G: Problem solving treatment and group psychoeducation for depression: multicentre randomised controlled trial. Outcomes of Depression International Network (ODIN) Group. BMJ 2000, 321(7274):1450-1454.

21. Terluin B, Van Heest FB, van der Meer K, Neomagus GJH, Hekman J, Aulbers LPJ, Starreveld JS, Grol MH: NHG-Standaard Angststoornissen (eerste herziening). Huisarts Wet 2007, 47(1):26-37.

22. van Marwijk H, Grundmeijer HGLM, Bijl D, van Gelderen MG, De Haan M, van Weel-Baumgarten EM, Burgers JS, Boukes FS, Romeijnders ACM: NHG-Standaard Depressieve stoornis (depressie). Huisarts Wet 2003, 46(11):614-622.

23. Zigmond AS, Snaith RP: The hospital anxiety and depression scale. Acta Psychiatr Scand 1983, 67(6):361-370.

24. EuroQol Group: EuroQol-a new facility for the measurement of health-related quality of life. The EuroQol Group. Health Policy 1990, 16(3):199-208.

25. Lamers LM, Stalmeier PF, McDonnell J, Krabbe PF, Van Busschbach JJ: Measuring the quality of life in economic evaluations: the Dutch EQ-5D tariff. Ned Tijdschr Geneeskd 2005, 149(28):1574-1578.

26. Hakkaart-van Roijen L, Van Straten A, Donker M, Tiemens B: Handleiding Trimbos/iMTA questionnaire for Costs associated with Psychiatric illness (TiC-P). Rotterdam; 2002

27. Oostenbrink JB, Koopmanschap MA, Rutten FF: Standardisation of costs: the Dutch Manual for Costing in economic evaluations. PharmacoEconomics 2002, 20(7):443-454.

28. Oostenbrink JB, Bouwmans CAM, Koopmanschap MA, Rutten FFH: Handleiding voor kostenonderzoek: Methoden en standaard kostprijzen voor economische evaluaties in de gezondheidszorg. Geactualiseerde versie 2004 [Handbook for cost studies: methods and standard costs for economic evaluation in health care. Updated version 2004]. Den Haag, The Netherlands: College voor Zorgverzekeringen; 2004.

29. Z-index: G-Standaard. The Hague, The Netherlands: Z-index; 2006.

30. Statistics Netherlands: Voorburg/Heerlen: Centraal Bureau voor de Statistiek (CBS); 2012.

31. Rubin DB: Multiple imputation for nonresponse in surveys. New York: John Wiley \& Sons; 1987.
32. Burton A, Billingham $\sqcup$, Bryan S: Cost-effectiveness in clinical trials: using multiple imputation to deal with incomplete cost data. Clin Trials 2007, 4(2):154-161.

33. Efron B: Missing data, imputation and the bootstrap. J Am Stat Assoc 1994, 89(426):463-475.

34. Black WC: The CE plane: a graphic representation of cost-effectiveness. Med Decis Making 1990, 10(3):212-214.

35. Fenwick $E$, O'Brien BJ, Briggs A: Cost-effectiveness acceptability curves-facts, fallacies and frequently asked questions. Health Econ 2004 13(5):405-415

36. Goetzel RZ, Ozminkowski RJ, Sederer LI, Mark TL: The business case for quality mental health services: why employers should care about the mental health and well-being of their employees. J Occup Environ Med 2002, 44(4):320-330.

37. Stewart WF, Ricci JA, Chee E, Hahn SR, Morganstein D: Cost of lost productive work time among US workers with depression. JAMA 2003, 289(23):3135-3144.

38. Briggs A: Economic evaluation and clinical trials: size matters. BMJ 2000 321(7273):1362-1363.

39. Cape J, Whittington C, Buszewicz M, Wallace P, Underwood L: Brief psychological therapies for anxiety and depression in primary care: meta-analysis and meta-regression. BMC Med 2010, 8:38.

doi:10.1186/1471-2296-13-98

Cite this article as: Bosmans et al:: Cost-effectiveness of problem-solving treatment in comparison with usual care for primary care patients with mental health problems: a randomized trial. BMC Family Practice 2012 13:98.

\section{Submit your next manuscript to BioMed Central and take full advantage of:}

- Convenient online submission

- Thorough peer review

- No space constraints or color figure charges

- Immediate publication on acceptance

- Inclusion in PubMed, CAS, Scopus and Google Scholar

- Research which is freely available for redistribution 